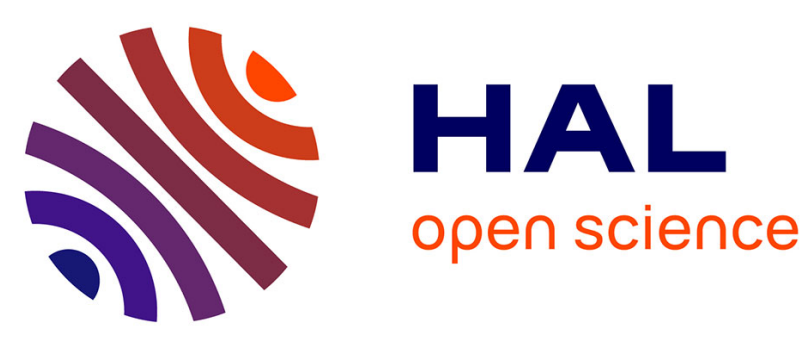

\title{
The adaptive value of probability distortion and risk-seeking in macaques' decision-making
}

Aurélien Nioche, Nicolas P. Rougier, Marc Deffains, Sacha Bourgeois-Gironde, Sébastien Ballesta, Thomas Boraud

\section{- To cite this version:}

Aurélien Nioche, Nicolas P. Rougier, Marc Deffains, Sacha Bourgeois-Gironde, Sébastien Ballesta, et al.. The adaptive value of probability distortion and risk-seeking in macaques' decision-making. Philosophical Transactions of the Royal Society B: Biological Sciences, 2021, 376 (1819), pp.20190668. 10.1098/rstb.2019.0668. hal-03005035v2

\section{HAL Id: hal-03005035 https://hal.inria.fr/hal-03005035v2}

Submitted on 25 Mar 2021

HAL is a multi-disciplinary open access archive for the deposit and dissemination of scientific research documents, whether they are published or not. The documents may come from teaching and research institutions in France or abroad, or from public or private research centers.
L'archive ouverte pluridisciplinaire HAL, est destinée au dépôt et à la diffusion de documents scientifiques de niveau recherche, publiés ou non, émanant des établissements d'enseignement et de recherche français ou étrangers, des laboratoires publics ou privés. 
The adaptive value of probability distortion and risk-seeking in macaques' decision-making

A. Nioche ${ }^{1, \dagger}$, N.P. Rougier $2,3,4,5, \dagger$, M. Deffains ${ }^{3,4}$, S. Bourgeois-Gironde ${ }^{6,7,8}$, S. Ballesta $^{9,10, \ddagger}, \mathrm{T}$. Boraud ${ }^{3,4,11, \ddagger}$, *

(1) Department of Communications and Networking, Aalto University, Finland, (2) Inria Bordeaux Sud-Ouest, 33405 Talence, France; (3) Institut des Maladies Neurodégénératives, Université de Bordeaux, 33000 Bordeaux, France; (4) Institut des Maladies Neurodégénératives, CNRS, UMR 5293, 33000 Bordeaux, France; (5) LaBRI, Université de Bordeaux, INP, CNRS, UMR 5800, 33405 Talence, France; (6) Laboratoire d'Economie Mathématique et de Microéconomie Appliquée, Université Panthéon-Assas, 75006 Paris, France; (7) Institut Jean Nicod, Département d'Etudes Cognitives, ENS, EHESS, PSL Research University, 75005 Paris, France; (8) Institut Jean Nicod, CNRS, UMR 8129; (9) Laboratoire de Neurosciences Cognitives et Adaptatives, UMR 7364, 67000 Strasbourg, France; (10) Centre de Primatologie de l'Université de Strasbourg, 67207 Niederhausbergen, France. (11) Centre Expert Parkinson, CHU Bordeaux, 33000 Bordeaux, France.

$\dagger$ These authors have contributed equally to this work.

$\ddagger$ These authors also have contributed equally to this work.

* Corresponding author: thomas.boraud@u-bordeaux.fr

Keywords: Genetic algorithm, Cognitive biases, Monkey, Autonomous Cognitive Testing, Experimental economics

\begin{abstract}
In humans, the attitude toward risk is not neutral and is dissimilar between bets involving gains and bets involving losses. The existence and prevalence of these decision features in non-human primates are unclear. In addition, only a few studies have tried to simulate the evolution of agents based on their attitude toward risk. Therefore, we still ignore to which extent Prospect theory's claims are evolutionary rooted. To shed light on this issue, we collected data in 9 macaques that performed bets involving gains or losses. We confirmed that their overall behaviour is coherent with Prospect theory's claims. In parallel, we used a genetic algorithm to simulate the evolution of a population of agents across several generations. We showed that the algorithm selects progressively agents that exhibit risk-seeking and an inverted S-shape distorted perception of probability. We compared these two results and found that monkeys' attitude toward risk when facing losses only is congruent with the simulation. This result is consistent with the idea that gambling in the loss domain is analogous to deciding in a context of life-threatening challenges where a certain level of risk-seeking behaviours and probability distortions may be adaptive.
\end{abstract}




\section{Introduction}

Making decisions with uncertain outcomes leads to solving a trade-off. A decisionmaker's attitude towards risk depends on his/her capacity to value quantities and perceive probabilities. Prospect theory offers a framework to define this attitude toward risk that relies on two key concepts: (i) choices are performed according to a reference point with respect to which expected gains and losses are contrasted; the evaluation of quantities is captured by the shape of the utility function and reference-dependence entails risk aversion in the domain of gains (concave utility function) and risk-seeking in the domain of losses (convex utility function). (ii) The perception of the probabilities of the outcomes, described by the shape of the probability weighting function, is non-linear.

An increasing number of studies have tackled the biological relevance of Prospect Theory claims [1-7]. In the domain of gains, risk aversion has been reported in several taxa, including rodents [8], birds [9], insects [10] and plants [11]. In apes and monkeys, a variable level of risk-seeking behaviour has been observed [12] and only few studies $[13,14]$ reported risk aversion (see [15], published in the same theme issue, for an exhaustive review of the literature in rhesus monkey [macaca mulatta]). Attitude toward risk in losses has seldom been reported in independent studies [15]. Prospect theory's claim of an asymmetry risk-aversion for gains/risk-seeking for losses per se has been assessed specifically in few studies [15]. An asymmetry similar to the one observed in humans has been reported in capuchins [16] and in Rhesus macaque [17] but other studies have not found any asymmetry for the latter [15]. Interestingly, this absence of asymmetry has also been reported in rodents [8]. Overall, these observed variability in primates' attitude toward risk can be partly explained by contextual factors or sampling effect [18-21].

Concerning probability distortion, the picture seems clearer. Rhesus and rats, for instance, perceive rare events as more probable than events that have a higher probability to occur and vice-versa $[8,17,22,23]$. This consistency across species, despite individual variability, possibly implies that probability distortion is likely to be anchored at a basic neurobiological level $[24,25]$, but more data need to be collected.

The patchy nature of the data collected up to now, concerning both the shape of the utility and probability functions in primates, calls for more comprehensive studies that assess the whole span of Prospect theory in larger populations in order to assess both intraindividual and interindividual variability, in more ecological conditions.

In addition, little is known about the adaptive value of the shape of the probability weighting function and the asymmetry of the utility function [26].

The goal of this study is therefore threefold: (i) to confirm the data previously collected with two rhesus macaques [17] using a larger number of animals, in more ecologically-valid conditions (i.e. semi-free ranging Tonkean macaques exposed to autonomous learning and testing devices); (ii) to address the adaptive 
value of the non-linearity of the utility and probability weighting function using a genetic algorithm-based simulation and (iii) to compare the above two findings.

\section{Materials and Methods}

\section{Ethics}

Experiments were conducted at the Centre de Primatologie (CdP) de l'Université de Strasbourg, (Niederhausbergen, France; LNCA UMR-7364) and Institut des Maladies Neurodegeneratives (IMN, CNRS, UMR-5293, University of Bordeaux, Bordeaux). At the University of Strasbourg, experiments were approved by the ethical committee of the Primate Centre of the University of Strasbourg (CdP), which is authorized to house non-human primates (registration $n^{\circ} \mathrm{B} 6732636$ ). The research further complied with the EU Directive 2010/63/EU for animal experiments. At the IMN, experimental procedures were performed in accordance with the Council Directive of 2010 (2010/63/EU) of the European Community and the National Institute of Health Guide for the Care and Use of Laboratory Animals. The protocol received agreement from the Ethical Committee for Animal Research CE50 (registration $n^{\circ} \mathrm{C} 33063268$ ).

\section{Subjects}

At the University of Strasbourg, we collected data on one social group of Tonkean macaques (Macaca tonkeana), housed at the CdP. Animals lived in semi-free ranging conditions in a wooded park of $3788 \mathrm{~m}^{2}$ with permanent access to an indoor-outdoor shelter $(2,5 \times 7.5 \mathrm{~m}-2 \times 4 \mathrm{~m})$. The group included 23 individuals with even sex ratio among adults, which is comparable to the composition of wild groups [27]. All subjects have research experiences in cognitive testing using touchscreens. Amongst the 23 individuals, 14 were involved in this current study, however only 7 were sufficiently trained or performed our economic task without a significant side bias $(<80 \%)$ to be included in this report. The species Macaca tonkeana is a member of a group of closely-related Sulawesi macaque species, all living in multi-male and multi-female egalitarian and highly tolerant societies $[28,29]$. Indeed, compared to other species of macaques, Sulawesi macaques social interactions are more complex and more influenced by friendships than by dominance and kinships [29-33]. However, the non-social cognition skills of Tonkean macaques seem to be comparable to those observed in other species of macaques [34]. Unfortunately, due to demographic heterogeneity and small size of the experimental population for each species (see Table S1), the comparison of economic decisions of Tonkean macaques with rhesus monkeys is beyond the scope of this actual study. However, we believe that the experimental methods reported here are particularly adapted to the future study of potential variations in economic decision-making in different species of primates. Water was provided ad libitum and monkeys were fed with commercial primate pellets twice a day and received fresh fruit and vegetables once a week. At the IMN, the study was performed on two female Rhesus macaques: Hav (born in 2012) and Gla (born in 2011). Animals were housed in the animal facility of the IMN under standard conditions (a 12h light/dark cycle with the light on from 7 am to $7 \mathrm{pm}$; humidity 
at $60 \%$, temperature $22 \pm 2^{\circ} \mathrm{C}$ ). During the time of the experiment, animals had controlled access to water five days per week. They were fed with commercial primate pellets once a day, and received fresh fruit and vegetables once a week. The 9 animals ( 5 males and 4 females) that composed the final experimental cohort weighed $10 \pm 3.1 \mathrm{~kg}$ and were $6.8 \pm 2$ years old.

\section{Data collection}

At the University of Strasbourg, data were collected using four Machines for Automated Learning and Testing (MALT), which were directly accessible to the monkeys from their living environment (Fig1a,b). Several cognitive tasks were available to the macaques at the MALT presented via a touchscreen interface [35]. Each MALT was accessible freely 24/7, except for two-hours cleaning and refill sessions, at least once a week. MALT allows automatic identification of each subject using an RFID dual-detection system [36]. For that purpose, subjects were all equipped with two RFID microchips (UNO MICRO ID / 12, ISO Transponder $2.12 * 12 \mathrm{~mm}$ ), injected into each forearm during the macaques' veterinary health check under appropriate anaesthesia, to individually identify them when using MALT (Fig1bc). At the IMN, data were daily collected $(\sim 1 \mathrm{~h} /$ session, $\sim 5$ sessions/week). During each session, animals were seated in a primate restraint chair located in a dark room equipped with a video monitoring system and faced a touchscreen on which the task was displayed (Fig1d). A resting bar was mounted in the lower part of the chair at waist level to accurately measure arm-reaching movement parameters. The behavioural and video data were stored on a separate computer located outside the room for further analysis.

Each monkey performed between 11,681 and 51,853 trials. Some semi-free ranging subjects (Tonkeans) were not performing the task optimally and adopted alternative response strategies. Indeed, five tested individuals chose the target on one side in more than $80 \%$ of the cases (in lotteries involving either gains or losses) and were consequently not included in this study. Two other individuals were also not considered because they performed less than 2,000 trials. Concerning the Rhesus monkeys, we recorded overall 54904 trials of which $39853(72.59 \%)$ had already been used for a previous publication [17].

\section{Task}

The task is adapted from Nioche et al. [17], without any major change and is shown in Fig2.

Lottery: We consider simple lotteries $L \in \mathcal{L}$, that can be defined as a tuple $(x, p)$, such that if $L=(x, p)$, it yields the outcome $x \in \mathbb{R}$, with probability $p \in[0,1]$ and 0 with probability $1-p$. For all trials, the probability of receiving (losing) tokens is drawn from the set $p \in\{0.25,0.50,0.75,1.00\}$.

Choice: Each choice has to be made between two lotteries $L_{1}=\left(\begin{array}{ll}x_{1} & p_{1}\end{array}\right)$ and $L_{2}=\left(\begin{array}{ll}x_{2} & p_{2}\end{array}\right)$.

Rewards: The monkey starts each trial with 3 tokens and can gain up to 3 tokens and lose up to 3 tokens. At the end of the trial, the monkey would be rewarded 
with liquid reward proportional to the tokens earned (between 0 and 6 tokens at the end of each trial). At the University of Strasbourg, monkeys could be rewarded with diluted syrup (1/10; 1 token of reward corresponds to $.25 \mathrm{~mL}$ of liquid reward; Fig2) and at the IMN, with diluted apple sauce $(1 / 3,1$ token corresponds to $.1 \mathrm{~mL}$ of liquid reward).

\section{Experimental paradigm}

At the beginning of the trial, a gauge with 3 tokens is displayed (Fig2bc). In Bordeaux, the monkey has to grasp a grip (i.e. resting bar at waist level) for a short duration that varies randomly in a range from trial to trial $(150 \sim 300 \mathrm{~ms})$ to ensure that the monkey can not anticipate the stimuli display. If the monkey does not hold the grip long enough, the trial is considered to be failed and an error is raised. If an error is raised, the screen turns to black, and the monkey has to wait 2,000 $\mathrm{ms}$ for the beginning of the next trial. In case the monkey holds the grip for the required amount of time, two circles representing two lotteries appear on the screen. The monkey has 2,000 ms to decide which lottery to choose. If the monkey does not choose within the allotted decision time, an error is raised. Once one lottery is selected by touching the corresponding circle, the other circle disappears. The monkey has $5,000 \mathrm{~ms}$ to return its hand to the grip (otherwise, an error is raised). Once the monkey returns its hand to the grip, the outcome is determined based on the probabilities shown in the two slices of the chosen circle. The amount of reward (loss) is indicated to the animal by the disappearance of the slice corresponding to the non-occurring output. The gauge is filled (emptied) by the amount earned (forfeited), one token at the time. The time of the filling animation is $1,500 \mathrm{~ms}$. The inter-trial interval varied randomly between 150 and $300 \mathrm{~ms}$.

At the University of Strasbourg, similar experiments were performed using touchscreens only. The experiment started with a central coloured square. Directly $(25 \mathrm{~ms})$ after the subject touched this cue, two circles representing two lotteries appeared on the screen. The animal had 15,000 ms to decide which lottery to choose. After making a choice by touching one of the two circles the other circle disappeared. On the remaining circle, the outcome was determined based on the probabilities shown in the two slices of the chosen circle. The gauge filled (emptied) by the amount earned (forfeited), all tokens at the time. During reward delivery, auditory feedback (bell sound) was played for each token earned by the subject. The gauge and tokens stayed on the screen for $8,000 \mathrm{~ms}$ plus a $500 \mathrm{~ms}$ intertrial interval where the screen was left black.

\section{Modelling of monkeys' decisions}

We characterize the monkeys' decision-making using a model based on the Prospect Theory [26,37], similar to the one used in Nioche et al. [17].

Probability weighting function and probability distortion. Following Prelec [38] the subjective probability perception is defined as:

$$
w(p)=e^{-(-\ln p)^{\alpha}}
$$


with $p \in(0,1]$ the actual probability, and with $\alpha \in(0, \infty)$, a free parameter indicating the distortion of the probability perception. We assume that $w(0)=0$. For $\alpha \in(0,1)$, the closer $\alpha$ is to zero, the more the small probabilities are overestimated, and the large probabilities underestimated. For $\alpha=1$, the subjective probabilities are the same as the objective probabilities. For $\alpha \in(1, \infty)$, the higher $\alpha$, the more the small probabilities are underestimated, and the large probabilities overestimated.

Utility function and risk aversion. The utility of a normalized outcome $x \in[-1,1]$ is defined as:

$$
u(x)= \begin{cases}x^{1-\beta} & \text { if } x>0 \\ -|x|^{1+\beta} & \text { if } x<0 \\ 0 & \text { otherwise }\end{cases}
$$

with $\beta \in \mathbb{R}$ a free parameter describing the risk-aversion of the decision-maker [39]. If $\beta$ is positive, $u^{\prime \prime}$ is negative, indicating risk-averse preferences [40], if $\beta$ is negative, $u^{\prime \prime}$ is positive, indicating risk-seeking preferences, if $\beta$ is equal to 0 , $\forall x: u(x)=x$ and $u^{\prime \prime}(x)=0$, indicating risk-neutral preferences.

Subjective expected utility and side bias. The subjective expected utility of a lottery $L$ is given by:

$$
\operatorname{SEU}(L=(x, p))= \begin{cases}w(p) u(x)-\gamma & \text { if } \gamma<0 \text { and } L \text { is on the left, } \\ w(p) u(x)+\gamma & \text { if } \gamma>0 \text { and } L \text { is on the right, } \\ w(p) u(x) & \text { otherwise. }\end{cases}
$$

with $\gamma \in \mathbb{R}$, a free parameter describing to which extent the decision-maker is biased towards one side. If $\gamma=0$, the decision-maker is not biased. Otherwise, the higher $\gamma$, the more he is biased towards the right, the lower $\gamma$, the more he is biased towards the left.

Choice probability and stochasticity. We also assume that action selection is probabilistic: the option that has the highest subjective utility is chosen only with a probability greater than the other options, and not with certainty. We model this stochasticity with a classic softmax function [41] such as the probability of choosing the lottery $L_{i}$ is given by:

$$
p\left(\text { choice }=L_{i}\right)=\frac{1}{1+e^{-\frac{S E U\left(L_{i}\right)-S E U\left(L_{j}\right)}{\lambda}}}
$$

with $L_{j}$ the alternative option, and $\lambda \in(0, \infty)$ a free parameter describing to which extent decision-making is stochastic. The higher $\lambda$, the more the decision-making is stochastic.

\section{Data analysis}

We will consider separately the choices involving only potential gains $\left(x_{i \in 1,2}>0\right)$ and only potential losses $\left(x_{i \in 1,2}<0\right)$. Furthermore, we will distinguish two groups of choices. 
Group 1. There is a better response regardless of the risk attitude of the decisionmaker: there is $i, j \in\{1,2\}$ s.t. $p_{i} \geq p_{j}$ and $x_{i}>x_{j}$, or $p_{i}>p_{j}$ and $x_{i} \geq x_{j}$, (30 different pairs of lotteries for gains, 30 for losses ignoring the order/side of presentation - 60 otherwise);

Group 2. A trade-off between risk and potential gain/loss has to be done: there is $i, j \in\{1,2\}$ s.t. $p_{i}>p_{j}$ and $x_{i}<x_{j}$ (18 different pairs of lotteries for gains, 18 for losses ignoring the order/side of presentation---36 otherwise).

The choices from Group 1 are used as control and choices from Group2 to assess attitude towards risk.

Control 1: Performance assessment. Lottery pairs with a better response (Group $1)$ are used to assess the monkeys' performance. We consider specifically the cases where it exists $i, j \in\{1,2\}$ s.t.:

- [Same $p] p_{i}=p_{j}$ but $x_{i}>x_{j}$ in order to assess the discrimination of the quantities (12 different pairs of lotteries for gains, 12 for losses ignoring the order/side of presentation - 24 otherwise);

- [Same $x] x_{i}=x_{j}$ but $p_{i}>p_{j}$ in order to assess the discrimination of the probabilities (18 different pairs of lotteries for gains, 18 for losses ignoring the order/side of presentation - 36 otherwise).

We model the probability of choosing the right option depending on the difference of expected values using an ordinary sigmoid function:

$$
p(\Delta)=1 /\left(1+e^{-k\left(\Delta-x_{0}\right)}\right)
$$

with $\Delta$ the difference of expected values between the lottery on the right and the lottery on the left, $k$ the slope parameter, and $x_{0}$ the intercept parameter. We used a Levenberg-Marquardt algorithm (SciPy library) to optimize the parameters.

Control 2: Consideration of the difference between expected values when tradingoff between quantity and probability. Results for lottery pairs with a trade-off between quantity and probability (Group 2) are used to check if the frequency with which the riskiest option is chosen is dependent on the difference between the expected values of the safest option and of the riskiest option.

We model this relation using an ordinary sigmoid function:

$$
p(\Delta)=1 /\left(1+e^{-k\left(\Delta-x_{0}\right)}\right)
$$

with $\Delta$ the difference of expected values between the riskiest lottery and the safest lottery, $k$ the slope parameter, and $x_{0}$ the intercept parameter. We used a Levenberg-Marquardt algorithm (SciPy library) to optimize the parameters.

Assessment of the attitude towards risk. The choices from Group 2 are used to characterize the attitude toward risk. To this end, separately for each monkey 
and for choices involving potential losses and for choices involving potential gains, we optimize the free parameters of our model $(\theta=\{\alpha, \beta, \gamma, \lambda\})$, using an SLSQP optimization algorithm [42] (Scipy Library), in order to maximize the likelihood of the data given the model. More precisely, the log-likelihood is estimated as follows:

$$
\ln \mathcal{L}(\mathcal{O} \mid \theta)=\sum_{i}^{|\mathcal{O}|} \ln p\left(o_{i} \mid \theta\right)
$$

for $\theta=\{\alpha, \beta, \gamma, \lambda\}$ a set of parameter values, and with $\mathcal{O}$ the set of observation under consideration, $p$ the probability according to our decision-making model of making the choice $o_{i} \in \mathcal{O}$ given $\theta$.

To assess the stability of the fit, we binned the trials by chunks of 200 trials by chronological order for each monkey, and optimize separately for each chunk the free parameters of the model.

\section{Simulations}

The simulation is based on a genetic algorithm [43]. We consider a set $L=$ $\left\{l_{1}, \ldots l_{N_{L}}\right\}$ of lotteries where each lottery $l_{1}$ is described by a probability $p_{i}=$ $i / N_{L}$ of reward and an associated reward $x_{i}=1 / p_{i}$ such that the expected value of each lottery is equal to 1 . We consider a set $A=\left\{a_{1}, \ldots, a_{N_{A}}\right\}$ of agents where each agent $a_{k}$ is fully described by a couple of parameters $\left(\alpha_{k}, \beta_{k}\right)$. When asked to choose between lotteries $l_{i}$ and $l_{j}$, the choice of agent $a_{k}$ is:

$$
\operatorname{choice}\left(a_{k}, l_{i}, l_{j}\right)= \begin{cases}i & \text { if } w_{\alpha_{k}}\left(p_{i}\right) u_{\beta_{k}}\left(x_{i}\right)>w_{\alpha_{k}}\left(p_{j}\right) u_{\beta_{k}}\left(x_{j}\right) \\ j & \text { otherwise }\end{cases}
$$

with $w_{\alpha}:[0,1] \rightarrow[0,1]$, the probability weighting function $w$ described above with a value of distortion parameter equal to $\alpha$ and $u_{\beta}: \mathbb{R} \rightarrow \mathbb{R}$, the utility function $u$ described above with risk aversion parameter equal to $\beta$.

The initial population $A^{0}$ is built from a set of parameters $\alpha$ and $\beta$ being uniformly drawn from $\left[\alpha_{\min }, \alpha_{\max }\right] \times\left[\beta_{\min }, \beta_{\max }\right]$ such that we have $a_{k}^{0}=\left\{\alpha_{k}, \beta_{k}\right\}$.

At each epoch, each agent completes a set of $N_{T}$ trials. Each trial is composed of two lotteries randomly and uniformly drawn for the set $L$. Individual gains are computed according to the agent's choices, and a proportion of the best scorers is selected using a selection rate $\gamma$ that may vary depending on the simulation (see results section). The next generation is computed by iteratively selecting two random parents among the selected agents and by computing the linear interpolation of their respective parameters $(\alpha$ and $\beta)$ such as to generate two offsprings. More precisely, considering an agent $a_{i}^{n}$ and an agent $a_{j}^{n}$ at epoch $n$, we generate two new agents $a_{k}^{n+1}$ and $a_{k+1}^{n+1}$ using a random and uniform factor $\lambda \in] 0, \varepsilon[$ ( $\varepsilon$ being the mixture rate) such that:

$$
\begin{aligned}
& a_{k}^{n+1}=\left(\lambda \alpha_{i}^{n}+(1-\lambda) \alpha_{j}^{n}, \lambda \beta_{i}^{n}+(1-\lambda) \beta_{j}^{n}\right) \\
& a_{k+1}^{n+1}=\left((1-\lambda) \alpha_{j}^{n}+\lambda \alpha_{i}^{n},(1-\lambda) \beta_{j}^{n}+\lambda \beta_{i}^{n}\right)
\end{aligned}
$$


The procedure is iterated until we reach a population whose size is the same as the initial population. After this new population has been generated, we apply a mutation of a small proportion of the new agents using a mutation rate $\delta$ such that $\delta \times N_{A}$ agents benefit from a random mutation. This mutation consists of replacing the agent's set of parameters by values randomly drawn from $\left[\alpha_{\min }, \alpha_{\max }\right] \times\left[\beta_{\min }, \beta_{\max }\right]$. The whole procedure is iterated for a fixed number of epochs $N_{E}$.

\section{Statistics}

To compare measures, we used a Wilcoxon signed-rank (for paired data) and rank-sum test (for independent data) with $p<0.05$. We also consider that monkeys' behaviours were significantly biased based on the $95 \%$ confidence intervals given by the best fit parameter value of the model.

\section{Results}

\section{Monkeys' attitude toward risk}

We analysed the results of 9 macaques monkeys that performed a total of 256976 trials (28 $553 \pm 12609$ per subject). Each trial consisted of a bet involving either gains or losses. We assessed the performance of the monkeys, by evaluating how they consider the difference of expected value between the available options. On average, monkeys were sensible to the difference of expected value between the two options. Supplementary section provides details about each individual behaviour (FigS1-S3 and Table S1-S8). Monkeys were sensible to the difference of expected value between the two options when probabilities were equal but amounts differ (Fig3a,d) for 8/9 individuals in the gain domain and $7 / 9$ in the loss domain ( $95 \%$ confidence intervals of the best fit parameter value). Monkeys were also sensible to options when amounts were equal but probability differs (Fig3b,e) for $9 / 9$ subjects in the gain and the loss domains and when there is a trading-off between quantity and probability (Fig3c,f), for 9/9 and 6/9 individuals for the gain and the loss domain, respectively.

Based on the Prospect theory $[26,37]$, we characterize the probability weighting function (i.e. subjective perception of probabilities), the utility function (i.e. subjective valuation of the rewards), the stochasticity in choice (i.e. to which extent choices reflect subjective expected utilities), and the side bias for each individual. On average, the best-fit values of the risk aversion parameter of the macaques' utility functions are significantly different for gains and losses (Wilcoxon signed-rank test, $\mathrm{p}=0.0039$ ). The recovered utility functions are respectively concave in the gains domain, indicating risk-averse preferences (Wilcoxon signed-rank test, $\mathrm{p}=0.02, \mathbf{F i g} 4 \mathbf{a}$ ) and convex in the losses domain, indicating risk-seeking preferences (Wilcoxon signed-rank test, $\mathrm{p}=0.0039$, Fig4d), reproducing the known asymmetry. Monkeys also overweight small probabilities both in the losses and gains domains (Fig4b,e; Wilcoxon signed-rank test, both $\mathrm{p}=0.0039$ ). This distortion is slightly more pronounced for losses than gains but did not reach a significant threshold (Wilcoxon signed-rank test, $\mathrm{p}=0.098$ ). The

steepness of the softmax function that fit their choice probabilities given the 
subjective expected utilities (Fig4e,f) was not significantly different between the gain and the loss domain (Wilcoxon signed-rank test, $\mathrm{p}=0.36$ ). The side bias in their decisions was not significantly different between the gain and the loss domain (Wilcoxon signed-rank test, $\mathrm{p}=0.91$ ). Supplementary section provides details about each individual (FigS4-S5).

\section{Evolution of attitude toward risk in a population of artificial agents}

Unless stated otherwise, we use the parameters given in Table 1 for all the simulations. The initial and final populations are respectively depicted in Fig5a,b and Fig5c,d. The thick black lines indicate the mean probability weighting function (Fig5c) and the mean utility function (Fig5d) over the whole population at the end of the selection process.

Overall, Fig 5 reveals that more than $95 \%$ of the agents in the final population tend to overestimate low probabilities and under-estimate high probabilities (see FigS6c). It also appears that more than $95 \%$ of the agents in the final population have a convex utility function, indicating risk-seeking behaviour (see FigS6d).

The mean gain of a subset of the artificial agents depending on the shapes on their probability weighting and utility functions is depicted in Fig6. The mean gain of best individuals is sometimes better than the expected value, allowing the selection process to be efficient. As expected, an overestimation of small probabilities and underestimation of high probabilities $(\beta=0.6)$ leads to higher gains with only a marginal influence of the utility. When there is no distortion of probabilities $(\beta=1.0)$, only the utility function influences the mean gain of the luckiest agents. For an underestimation of small probabilities and overestimation of high probabilities $(\beta=1.4)$, the influence of the utility function is negligible.

The exact shape of the final population's functions is dependent on the selection rate, mutation rate and mixture rate as shown in Fig7. However, the influence of the mutation rate is small, as is the influence of the mixture rate, and for neighbouring values of the selection rate (between $5 \%$ and $30 \%$ ), the shapes of the curves remain similar to the ones shown in Fig5. Though, if the selection rate is very large ( $45 \%$ or higher), the shapes of the curves are inverted: the probability weighting function would be S-shaped (instead of inverted S-shaped), indicating a low probabilities underestimation and a high probabilities overestimation, and the utility function would be convex, indicating risk-averse preferences. Further analysis of the evolution and influence of parameters on the distribution of the final population are provided in Figures S7-13.

\section{Comparison between monkeys' and agents' populations}

We compared the observations made in monkeys and the observations made in artificial agents. Fig8a,b summarizes the results of this analysis.

As the exact characteristics of the final population depend on the selection rate, we report statistical results for a selection parameter included in the set $\{0.05,0.1, \ldots, 0.35\}$, that is values neighbouring the one used for the results 
presented in Fig5 $( \pm 0.15)$. The probability weighting function has an inverted S-shape both in populations of monkeys and selected artificial agents. The values of the probability distortion parameter between the populations of monkeys and selected artificial agents are not significantly different for gains (Fig8b, Wilcoxon rank-sum, all p>0.05, common language effect size [44], $\mathrm{f}=[0.32,0.58])$ and less robustly for losses (Fig8b, Wilcoxon rank-sum, for $\gamma \in[0.05,0.2]$, all p $>0.05$, $\mathrm{f}=[0.49,0.63]$ and for $\gamma \in[0.25,0.35]$, all $\mathrm{p}<0.05, \mathrm{f}=[0.12,0.29])$. On the other hand, the values of the risk aversion parameter are significantly different for gains (Fig8b, Wilcoxon rank-sum, all $\mathrm{p}<0.001, \mathrm{f}=[0.91,1])$ and, to a lesser extent, for losses (Fig8b, Wilcoxon rank-sum, all $p<0.1, f=[0.12,0.32]$ ), although the values observed for monkeys in the losses and artificial agents lead both to risk-seeking preferences (convexity of the utility function).

\section{Discussion}

We measured probability distortion and risk aversion in nine macaque monkeys of different ages and genders, belonging to two species, by using a similar task in different experimental conditions. We found that, overall, monkeys showed an inverted S-shape probability distortion pattern. In addition, on average, animals were respectively risk-averse for gains and risk-seeking for losses, confirming an asymmetry of treatment between gains and losses as we previously described [17]. Our conclusions are drawn from a dataset that includes a reasonable number of subjects and most of them voluntarily performed the task in unconstrained environments, providing ecological validity to our findings. Our results therefore reliably reproduce in two species of non-human primates the classical pattern of the Prospect theory found in humans [45]. It is still worth noting that a substantial level of heterogeneity has been reported in the attitude of monkeys and humans towards risk [21] (for more information see [15] published in this special issue). Many confounding factors have been considered as the source of this heterogeneity, such as the amount or the nature of rewards, the kind of behavioural task or even the temporal organization of trials within a given task. Further research is needed to better understand the influence of these factors on primates' decision-making.

Efficiency in foraging and reproduction has been optimized by natural selection as individuals that followed sub-optimal strategies lost out to competitors $[46,47]$. The classical inverted S-shape profile of the probability weighting function has been described in many different species, and it may be a ubiquitous characteristic of decision-making of living organisms [8,22,23]. One can thus speculate that such decisional strategy may be adaptive. The evolutionary advantages of the non-linearity of the probability weighting and utility functions remain debated [1-7]. Our results, that bring together real-life and simulation data, offer new insights into this issue.

Indeed, to assess if these cognitive biases may confer an evolutionary advantage at the population level, we ran a simulation that allowed agents to freely compete and select the decision strategies that had the best fitness. The results of this simulation show that the final population exhibits an inverted S-shaped 
probability weighting function and a convex utility function. This indicates that the selected decision-makers are those that have a preference for risk (i.e. the convexity of the utility function) while overestimating the small probabilities and underestimating the high probabilities (i.e. the inverted S-shape of the probability weighting function). Hence, in a context where the total number of choices is limited, and the expected value is constant between the options, the selection process promotes the lucky gamblers that are biased towards the 'high-stake' bets (small probability and large reward). The strategy with the better fitness in our simulation is therefore congruent with the behavioural pattern of the monkeys' behaviour in losses.

Gambling in the loss domain can be arguably considered analogous to deciding in a context of life-threatening challenges (e.g. predation avoidance, social challenges). Cognitive adaptation in humans seems to support this hypothesis [48]. For instance, loss cues attracted more attentional resources than gain cues $[49,50]$. In real-life, suboptimal decision-making in the gains domain may thus not lead to consequences as dramatic as in the losses domain. Hence, one can speculate that different levels of selective pressure could have been applied to the biological mechanisms responsible for decision-making under risk in the gain and the loss domains, then fostering an asymmetry of treatment.

Our simulation did not capture this known asymmetry of treatment between gains and losses in primates [26,37]. This was expected because, for agents in the simulation, gains and losses were differentiated only by their polarity. However, the mean decisional strategy adopted by monkeys in the losses' domain was the closest to the selected behaviors of agents (both risk seeking). Several proposals may help to interpret this result. First, satiety is known to influence economic decisions [51-54]. In real-life, losses could be arguably infinite (i.e. losing social status, territories, physical integrity or even facing death) whereas accumulation of goods is usually limited by time and/or space (e.g. limited accumulation of goods or satiety during foraging). These features were not implemented in the simulation and may be good candidates to better explain the known asymmetry of treatment between gains and losses. Second, it has been noted that the emotional state of subjects and/or social context of decisions can influence attitude toward risk and loss aversion $[19,55,56]$. Our simulations did not consider these other important features of biological agents. But despite these limits, we found that the final population of agents exhibit distorted probability perception and non-linear utility function, thus showing that these behavioural strategies can be adaptive in a given context of decision-making. In conclusion, our study shows that integrating simulation and real-life data provides new insights about the evolutionary roots of cognitive biases, therefore reassessing the biological dimension of the decision theory. 


\section{References}

1. Okasha S. 2007 Rational Choice, Risk Aversion, and Evolution. The Journal of Philosophy 104, 217-235.

2. McDermott R, Fowler JH, Smirnov O. 2008 On the Evolutionary Origin of Prospect Theory Preferences. The Journal of Politics 70, 335-350. (doi:10.1017/S0022381608080341)

3. Houston AI, Fawcett TW, Mallpress DEW, McNamara JM. 2014 Clarifying the relationship between prospect theory and risk-sensitive foraging theory. Evolution and Human Behavior 35, 502-507. (doi:10.1016/j.evolhumbehav.2014.06.010)

4. Rieger MO. 2014 Evolutionary stability of prospect theory preferences. Journal of Mathematical Economics 50, 1-11. (doi:10.1016/j.jmateco.2013.11.002)

5. Hintze A, Olson RS, Adami C, Hertwig R. 2015 Risk sensitivity as an evolutionary adaptation. Sci Rep 5, 8242. (doi:10.1038/srep08242)

6. Mallpress DEW, Fawcett TW, Houston AI, McNamara JM. 2015 Risk attitudes in a changing environment: An evolutionary model of the fourfold pattern of risk preferences. Psychological Review 122, 364-375. (doi:10.1037/a0038970)

7. Addessi E, Beran MJ, Bourgeois-Gironde S, Brosnan SF, Leca JB. 2020 Are the roots of human economic systems shared with nonhuman primates? Neuroscience \&s Biobehavioral Reviews 109, 1-15. (doi:10.1016/j.neubiorev.2019.12.026)

8. Constantinople CM, Piet AT, Brody CD. 2019 An Analysis of Decision under Risk in Rats. Current Biology 29, 2066-2074.e5. (doi:10.1016/j.cub.2019.05.013)

9. Caraco T. 1981 Energy budgets, risk and foraging preferences in dark-eyed juncos (Junco hyemalis). Behav Ecol Sociobiol 8, 213-217. (doi:10.1007/BF00299833)

10. Harder LD, Real LA. 1987 Why are Bumble Bees Risk Averse? Ecology 68, 1104-1108. (doi:10.2307/1938384)

11. Dener E, Kacelnik A, Shemesh H. 2016 Pea Plants Show Risk Sensitivity. Current Biology 26, 1763-1767. (doi:10.1016/j.cub.2016.05.008)

12. Sayers K, Menzel CR. 2017 Risk sensitivity, phylogenetic reconstruction, and four chimpanzees. Behav Ecol Sociobiol 71, 26. (doi:10.1007/s00265-016-2234-8)

13. Heilbronner SR, Rosati AG, Stevens JR, Hare B, Hauser MD. 2008 A fruit in the hand or two in the bush? Divergent risk preferences in chimpanzees and bonobos. Biol. Lett. 4, 246-249. (doi:10.1098/rsbl.2008.0081)

14. Eisenreich BR, Hayden BY, Zimmermann J. 2019 Macaques are risk-averse in a freely moving foraging task. Sci Rep 9, 15091. (doi:10.1038/s41598-01951442-z) 
15. Garcia B, Cerrotti F, Palminteri S. 2020 The description-experience gap: a challenge for the neuroeconomics of decision-making under uncertainty. (doi:10.31234/osf.io/9s23f)

16. Lakshminarayanan VR, Chen MK, Santos LR. 2011 The evolution of decision-making under risk: Framing effects in monkey risk preferences. Journal of Experimental Social Psychology 47, 689-693. (doi:10.1016/j.jesp.2010.12.011)

17. Nioche A, Bourgeois-Gironde S, Boraud T. 2019 An asymmetry of treatment between lotteries involving gains and losses in rhesus monkeys. Sci Rep $\mathbf{9}, 10441$. (doi:10.1038/s41598-019-46975-2)

18. Farashahi S, Azab H, Hayden B, Soltani A. 2018 On the Flexibility of Basic Risk Attitudes in Monkeys. J. Neurosci. 38, 4383-4398. (doi:10.1523/JNEUROSCI.2260-17.2018)

19. Rosati AG, Hare B. 2012 Decision making across social contexts: competition increases preferences for risk in chimpanzees and bonobos. Animal Behaviour 84, 869-879. (doi:10.1016/j.anbehav.2012.07.010)

20. De Petrillo F, Paoletti M, Bellagamba F, Manzi G, Paglieri F, Addessi E. 2020 Contextual factors modulate risk preferences in adult humans. Behavioural Processes 176, 104137. (doi:10.1016/j.beproc.2020.104137)

21. Harrison GW, Elisabet Rutström E. 2008 Risk Aversion in the Laboratory. In Risk Aversion in Experiments (eds J C. Cox, G W. Harrison), pp. 41-196. Emerald Group Publishing Limited. (doi:10.1016/S0193-2306(08)00003-3)

22. Stauffer WR, Lak A, Bossaerts P, Schultz W. 2015 Economic Choices Reveal Probability Distortion in Macaque Monkeys. Journal of Neuroscience 35, 3146-3154. (doi:10.1523/JNEUROSCI.3653-14.2015)

23. Ferrari-Toniolo S, Bujold PM, Schultz W. 2019 Probability Distortion Depends on Choice Sequence in Rhesus Monkeys. J. Neurosci. 39, 2915-2929. (doi:10.1523/JNEUROSCI.1454-18.2018)

24. Tobler PN, Christopoulos GI, O'Doherty JP, Dolan RJ, Schultz W. 2008 Neuronal Distortions of Reward Probability without Choice. Journal of Neuroscience 28, 11703-11711. (doi:10.1523/JNEUROSCI.2870-08.2008)

25. Wu S-W, Delgado MR, Maloney LT. 2011 The Neural Correlates of Subjective Utility of Monetary Outcome and Probability Weight in Economic and in Motor Decision under Risk. J. Neurosci. 31, 8822-8831. (doi:10.1523/JNEUROSCI.0540-11.2011)

26. Tversky A, Kahneman D. 1992 Advances in prospect theory: Cumulative representation of uncertainty. J Risk Uncertainty 5, 297-323. (doi:10.1007/BF00122574)

27. Riley EP. 2007 Flexibility in Diet and Activity Patterns of Macaca tonkeana in Response to Anthropogenic Habitat Alteration. Int J Primatol 28, 107-133. (doi:10.1007/s10764-006-9104-6) 
28. Thierry B, Iwaniuk AN, Pellis SM. 2000 The Influence of Phylogeny on the Social Behaviour of Macaques (Primates: Cercopithecidae, genus Macaca). Ethology 106, 713-728.

29. Thierry B, Bynum EL, Baker S, Kinnaird MF, Matsumura S, Muroyama Y, O'Brien TG, Petit O, Watanabe K. 2000 The Social Repertoire of Sulawesi Macaques. Primate Research 16, 203-226. (doi:10.2354/psj.16.203)

30. Petit O, Thierry B. 1994 Aggressive and peaceful interventions in conflicts in Tonkean macaques. Animal Behaviour 48, 1427-1436. (doi:10.1006/anbe.1994.1378)

31. Dobson SD. 2012 Coevolution of Facial Expression and Social Tolerance in Macaques. American Journal of Primatology 74, 229-235. (doi:10.1002/ajp.21991)

32. Micheletta J, Waller BM. 2012 Friendship affects gaze following in a tolerant species of macaque, Macaca nigra. Animal Behaviour 83, 459-467. (doi:10.1016/j.anbehav.2011.11.018)

33. Whitehouse J, Meunier H. 2020 An understanding of third-party friendships in a tolerant macaque. Scientific Reports 10, 9777. (doi:10.1038/s41598-020$66407-w)$

34. Joly M, Micheletta J, De Marco A, Langermans JA, Sterck EHM, Waller BM. 2017 Comparing physical and social cognitive skills in macaque species with different degrees of social tolerance. Proceedings of the Royal Society B: Biological Sciences 284, 20162738. (doi:10.1098/rspb.2016.2738)

35. Fizet J, Rimele A, Pebayle T, Cassel J-C, Kelche C, Meunier H. 2017 An autonomous, automated and mobile device to concurrently assess several cognitive functions in group-living non-human primates. Neurobiol Learn Mem 145, 45-58. (doi:10.1016/j.nlm.2017.07.013)

36. Pebayle T, Fizet J, Rimele A, Meunier H. 2016 Multitask learning machine with dual RFID detection.

37. Kahneman D, Tversky A. 1979 Prospect Theory: An Analysis of Decision under Risk. Econometrica 47, 263-291. (doi:10.2307/1914185)

38. Prelec D. 1998 The Probability Weighting Function. Econometrica 66, 497-527. (doi:10.2307/2998573)

39. Holt CA, Laury SK. 2002 Risk Aversion and Incentive Effects. The American Economic Review 92, 1644-1655.

40. Von Neumann J, Morgenstern O. 1944 Theory of games and economic behavior. Princeton, NJ, US: Princeton University Press.

41. Sutton RS, Barto AG. 1998 Reinforcement learning: an introduction. Cambridge, Mass: MIT Press. 
42. Kraft D. 1988 A Software Package for Sequential Quadratic Programming. Wiss. Berichtswesen d. DFVLR.

43. Holland JH. 1992 Genetic Algorithms. Sci Am 267, 66-72.

(doi:10.1038/scientificamerican0792-66)

44. McGraw KO, Wong SP. 1992 A common language effect size statistic. Psychological Bulletin 111, 361-365. (doi:10.1037/0033-2909.111.2.361)

45. Ruggeri K et al. 2020 Replicating patterns of prospect theory for decision under risk. Nature Human Behaviour , 1-12. (doi:10.1038/s41562-020-0886-x)

46. Parker GA, Smith JM. 1990 Optimality theory in evolutionary biology. Nature 348, 27-33. (doi:10.1038/348027a0)

47. Darwin C Burrow, John Wyon. 1859 The origin of species by means of natural selection or the preservation of favoured races in the struggle for life. Harmondsworth; New-York; Ringwood: Penguin books.

48. Harinck F, Van Dijk E, Van Beest I, Mersmann P. 2007 When Gains Loom Larger Than Losses: Reversed Loss Aversion for Small Amounts of Money. Psychol Sci 18, 1099-1105. (doi:10.1111/j.1467-9280.2007.02031.x)

49. Lejarraga T, Schulte-Mecklenbeck M, Pachur T, Hertwig R. 2019 The attention-aversion gap: how allocation of attention relates to loss aversion. Evolution and Human Behavior 40, 457-469. (doi:10.1016/j.evolhumbehav.2019.05.008)

50. Sheng F, Ramakrishnan A, Seok D, Zhao WJ, Thelaus S, Cen P, Platt ML. 2020 Decomposing loss aversion from gaze allocation and pupil dilation. Proc Natl Acad Sci USA 117, 11356-11363. (doi:10.1073/pnas.1919670117)

51. Raghuraman AP, Padoa-Schioppa C. 2014 Integration of Multiple Determinants in the Neuronal Computation of Economic Values. Journal of Neuroscience 34, 11583-11603. (doi:10.1523/JNEUROSCI.1235-14.2014)

52. Sheng CL. 1989 Some quantitative concepts of value and utility from a utilitarian point of view. Theor Decis 26, 175-195. (doi:10.1007/BF00159225)

53. Yamada H, Tymula A, Louie K, Glimcher PW. 2013 Thirst-dependent risk preferences in monkeys identify a primitive form of wealth. PNAS 110, 15788-15793. (doi:10.1073/pnas.1308718110)

54. Yamada H. 2017 Hunger enhances consistent economic choices in non-human primates. Sci Rep 7, 2394. (doi:10.1038/s41598-017-02417-5)

55. Druckman JN, McDermott R. 2008 Emotion and the Framing of Risky Choice. Polit Behav 30, 297-321. (doi:10.1007/s11109-008-9056-y)

56. Campos-Vazquez RM, Cuilty E. 2014 The role of emotions on risk aversion: A Prospect Theory experiment. Journal of Behavioral and Experimental Economics 50, 1-9. (doi:10.1016/j.socec.2014.01.001) 


\section{Data availability}

The data necessary to reproduce the analyses presented in this article are provided at https://github.com/aureliennioche/EvoProspect.

\section{Code availability}

The code necessary to reproduce the simulations and the analyses presented in this article is provided at https://github.com/aureliennioche/EvoProspect.

\section{Acknowledgements}

We are grateful to Silabe and the University of Strasbourg for expert animal care of the Tonkean colony and financial support. We thank Hélène Meunier for the initial training of the Tonkean colony on a touchscreen interface and Adam Rimele for technical support and data management. The rhesus macaques were housed in the centre Paul Broca Nouvelle Aquitaine, thanks to Labex BRAIN financial support. Hugues Orignac and Tho-Hai N'Guyen take care of them with great skills. TB and MD are supported by the CNRS, SB by the University of Strasbourg, SBG by the University of Panthéon-Assas, NR by Inria and AN by the Agence Nationale de la Recherche (ANR-16-CE38-0003), the Ministère de la Recherche et de la Technologie (French ministry for research and technology), Sorbonne Université and Aalto University. The funders had no role in study design, data collection, and interpretation, or the decision to submit the work for publication.

\section{Author Contributions}

AN, MD, SB performed the experiments in monkeys; AN, NR performed the simulations; AN, NR and SB performed the data analysis; AN, NR, MD, SBG, $\mathrm{SB}$, and TB designed the study and co-wrote the manuscript.

\section{Competing Interests}

The authors declare that they have no competing interests. 

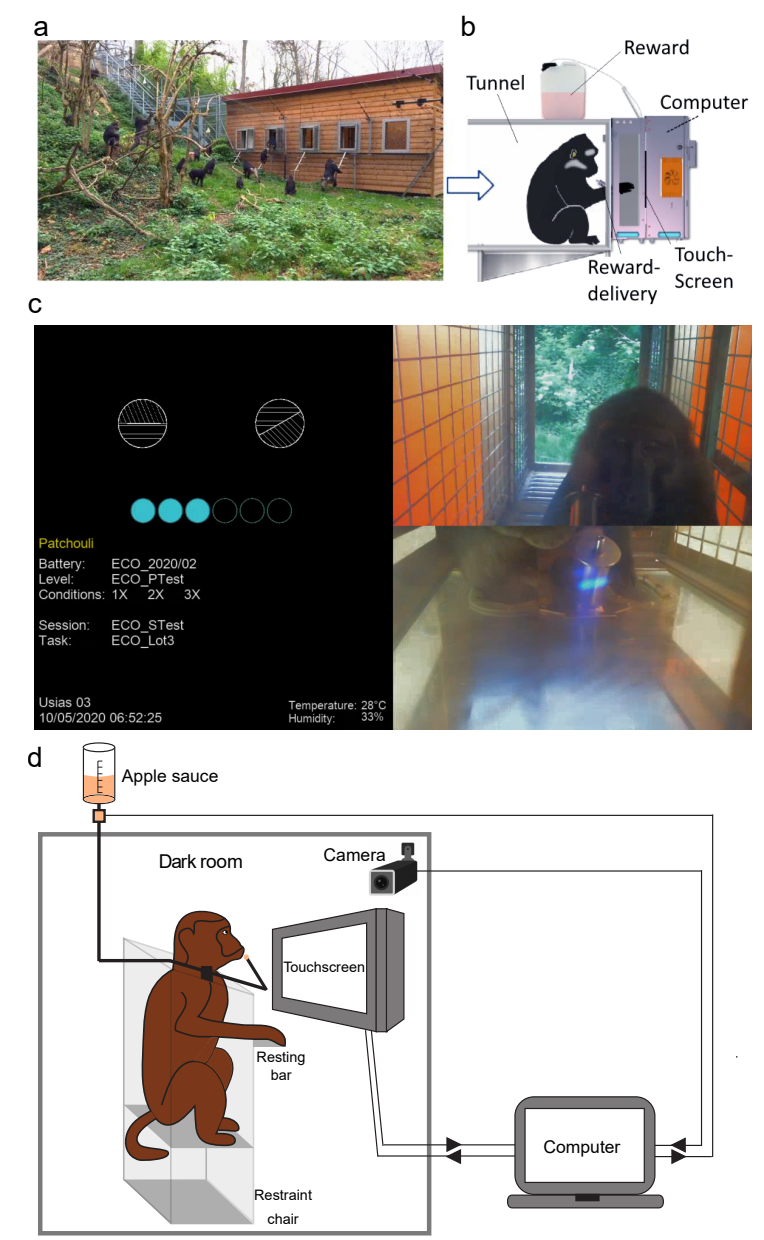

Figure 1: $(\mathbf{a}, \mathbf{b})$ Four MALTs were set up in a shelter placed inside the macaques' wooded park and behavioural tasks similar to the ones used in Nioche et al. [17] were presented via a touchscreen interface. Correct trials are rewarded with diluted syrups according to the probability associated with the choice. (c) Screenshot from the control video streaming while a subject was performing a trial. The pattern of each slice represents the number of tokens earned or lost (see Methods). (d) Experimental device for rhesus monkeys at the IMN. The monkey sat in a primate restraint chair positioned $20 \mathrm{~cm}$ from a touchscreen installed in an electrically isolated dark room. A resting bar was mounted on the lower part of the chair at waist level. A tube positioned directly in front of the monkey's mouth dispensed small amounts of apple sauce as a reward. Task/video monitoring and data acquisition were performed by a separate computer located outside the dark room. 


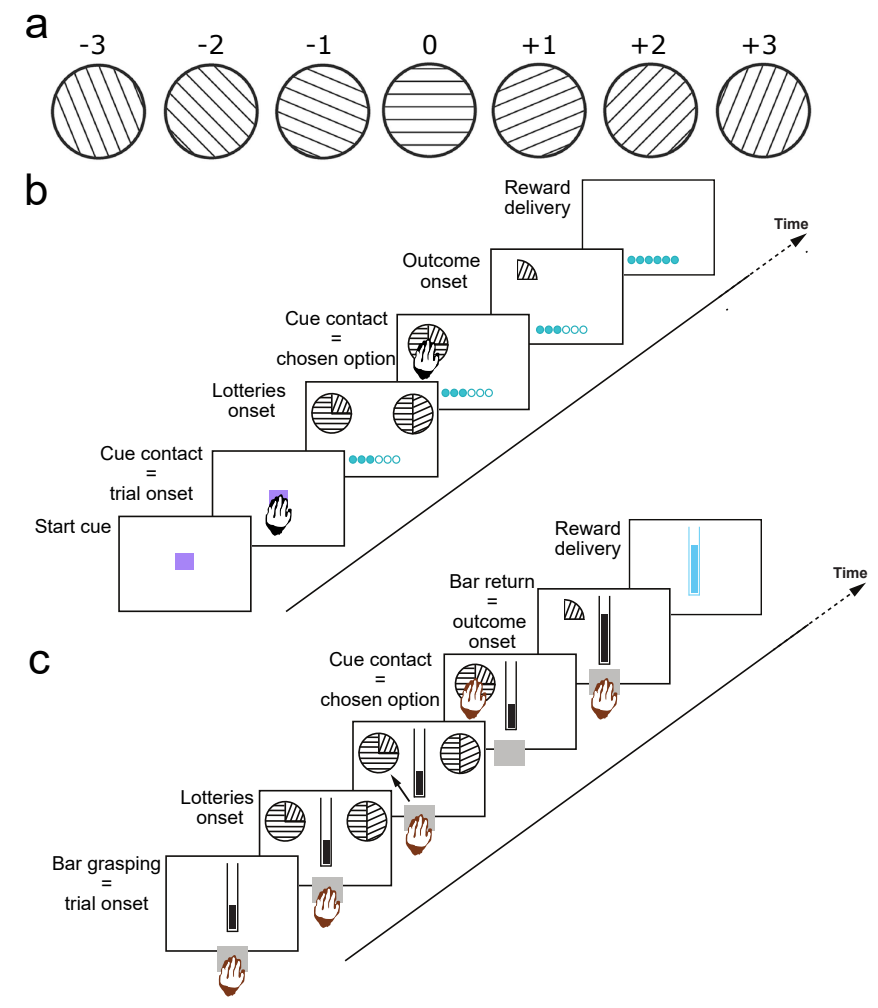

Figure 2: (a) The orientation of the parallel lines constituting the pattern indicates a quantity (horizontal lines represent 0 ; clockwise rotation of one-, two-, three-quarters of $90^{\circ}$ represent respectively a loss of $-1,-2,-3$ tokens; counterclockwise rotation of one-, two-, three-quarters of $90^{\circ}$ represent respectively a gain of 1, 2, 3 tokens). (b,c) Each lottery is represented by a pie chart, as in Stauffer et al. [22]. Each pie chart can be composed of two slices. Each slice encodes one possible outcome of the lottery $(x$ or 0$)$. The arc length of each slice represents the probability of the corresponding outcome $(p$ or $1-p)$. The relative positions on the pie chart of each slice are randomly determined at each trial. Panel b and c represents the task as it was coded at the University Strasbourg and the IMN of Bordeaux, respectively. 

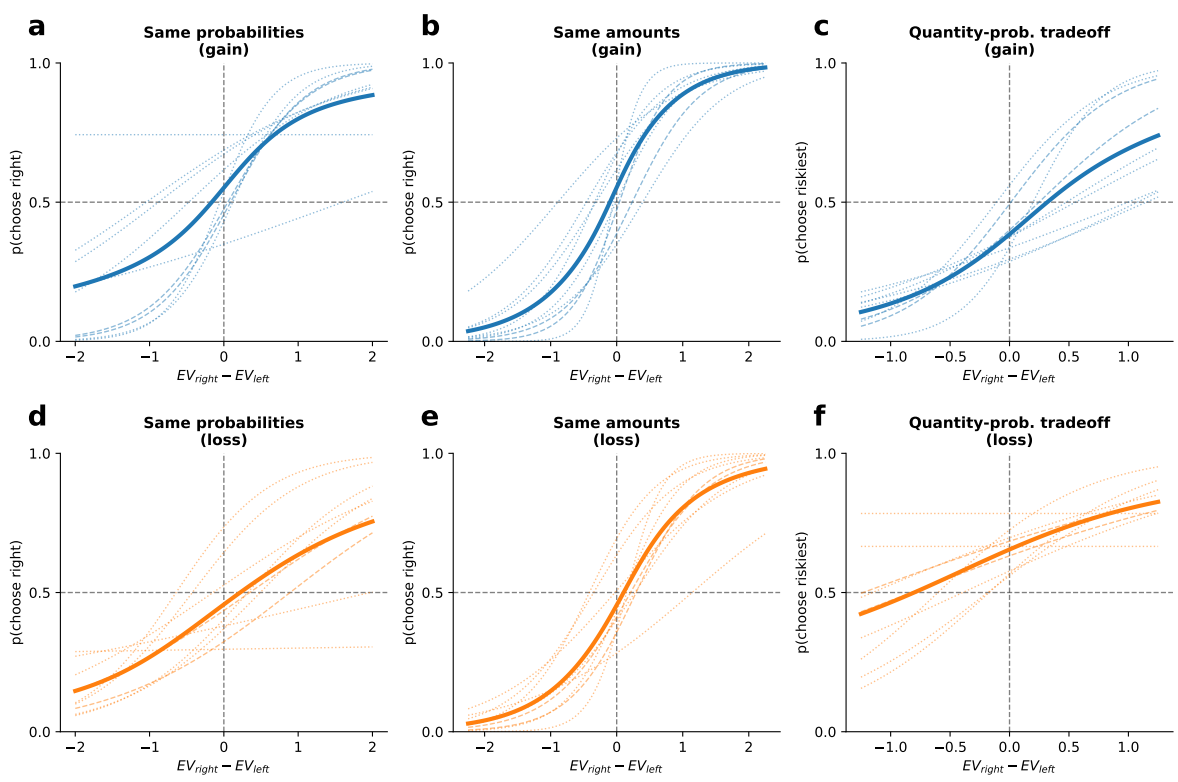

Figure 3: Consideration of the difference between expected values when probabilities are equal but amounts differ, when amounts are equal but probability differ, or when there is a trade-off between quantity and probability. (a,d) Probability of choosing the right option against the difference of EV when probabilities are equal but amounts differ. (b,e) Probability of choosing the right option against the difference of EV when amounts are equal but probability differ. (c,f) Probability of choosing the riskiest option against the difference of $\mathrm{EV}$ when there is a trading-off between quantity and probability. In all panels, blue lines are related to choices involving gains, and orange lines to choices involving losses. Thin lines represent the value of ordinary two parameters sigmoid functions using the best-fit parameter values of each individual (one line corresponds to one individual). Thick lines represent the average value of these functions. 

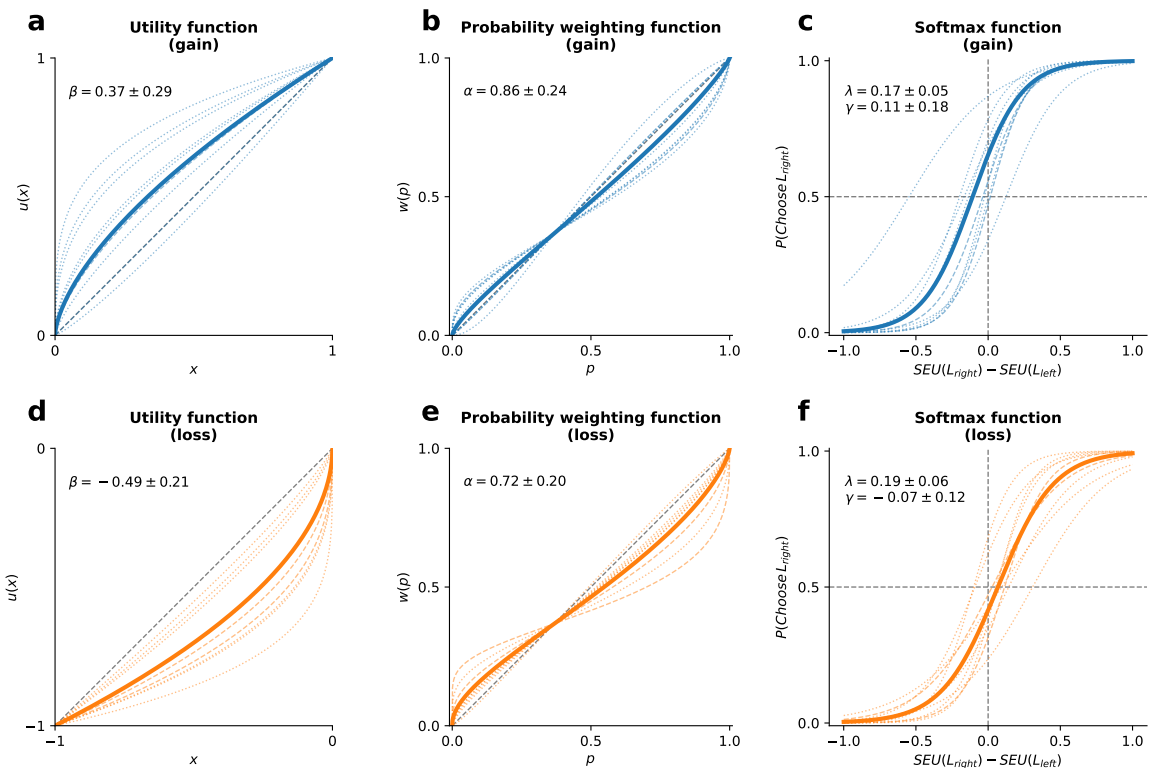

Figure 4: Modelling of monkeys' attitude toward risk. (a,d) Monkeys' utility function, (b,e) monkeys' probability weighting function, (c,f) monkeys' probability to choose the right lottery according to the difference of Subjective Expected Utility (SEU) between right and left lotteries. In all panels, blue lines are related to choices involving gains, and orange lines to choices involving losses. Thin lines represent the indicated function using the average best-fit parameter values over the data chunks of one individual, and thick lines represent the indicated function using the mean of the average best-fit parameter values over all the individuals. The mean value \pm STD) for each parameter for each condition is indicated in the left corner. 
a

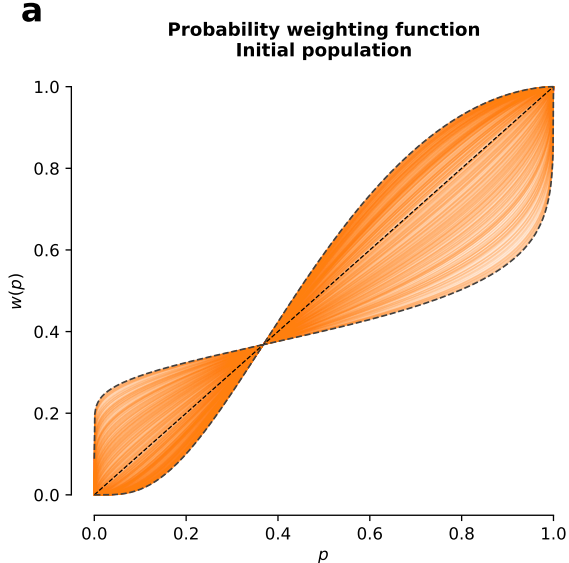

C

Probability weighting function

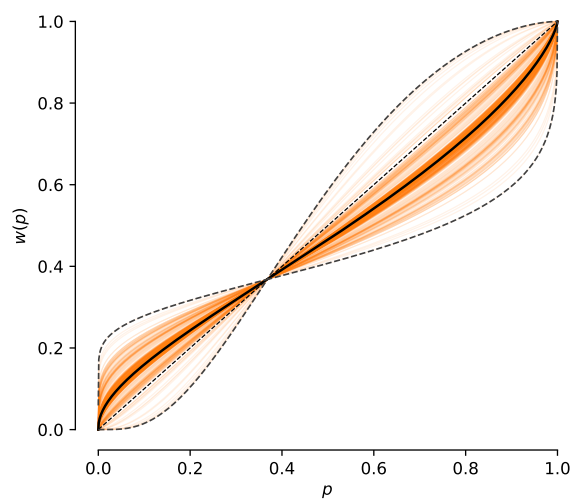

b

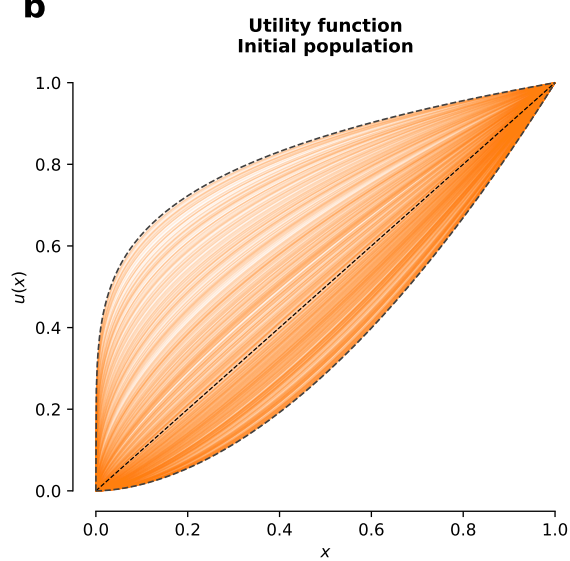

d
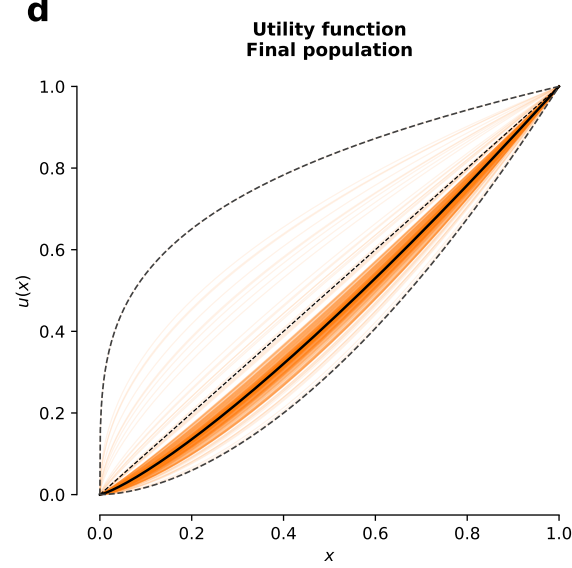

Figure 5: Simulation of 1000 agents playing 100 lotteries and over 1000 generations. (a,c) Probability weighting function. (b,d) Utility function. The initial population is generated using parameters $\left(\alpha_{k}, \beta_{k}\right)$ randomly and uniformly drawn from $\left[\alpha_{\min }, \alpha_{\max }\right] \times\left[\beta_{\min }, \beta_{\max }\right]$. The apparent asymmetry of initial curves comes from the non-linearity of parameter effects on function shapes. After 1000 epochs where we applied the selection of the best agents, generated new agents from their parameters and introduced a few random variations, the final mean behaviour of agents is shown using a thick black curve. 

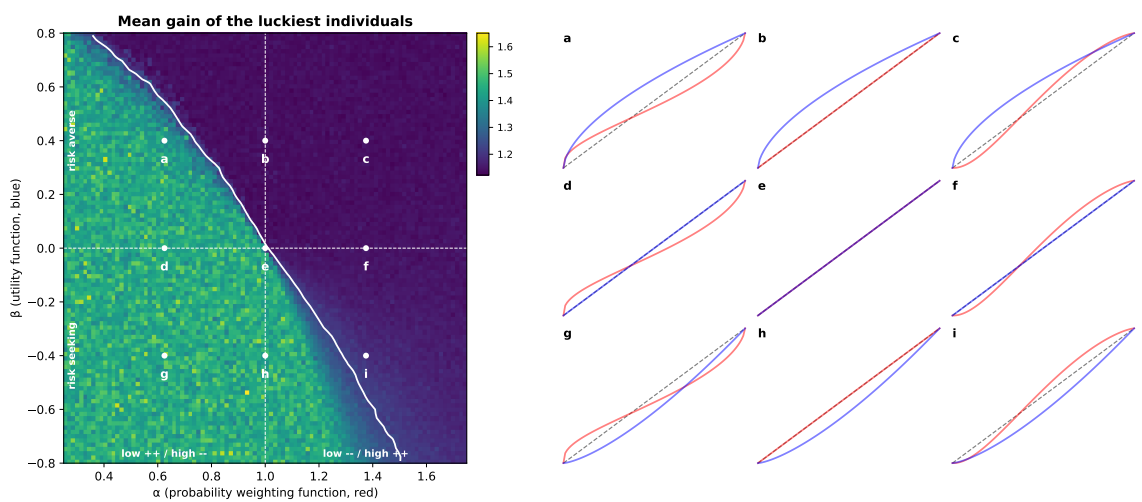

Figure 6: Analysis of the mean gain of a population of agents. Each point on the left panel corresponds to the mean gain of a subset $(20 \%$ on the figure) of a group of $N_{A}$ agents playing $N_{T}$ lotteries. Depending on their probability weighting and utility functions, the mean gain of best individuals might be better than the expected value. The white line corresponds to the separation along with the median score (1.23) of all simulations. On the right, we display the utility function (blue) and the probability weighting function (red) for a few representative points (a-i). 

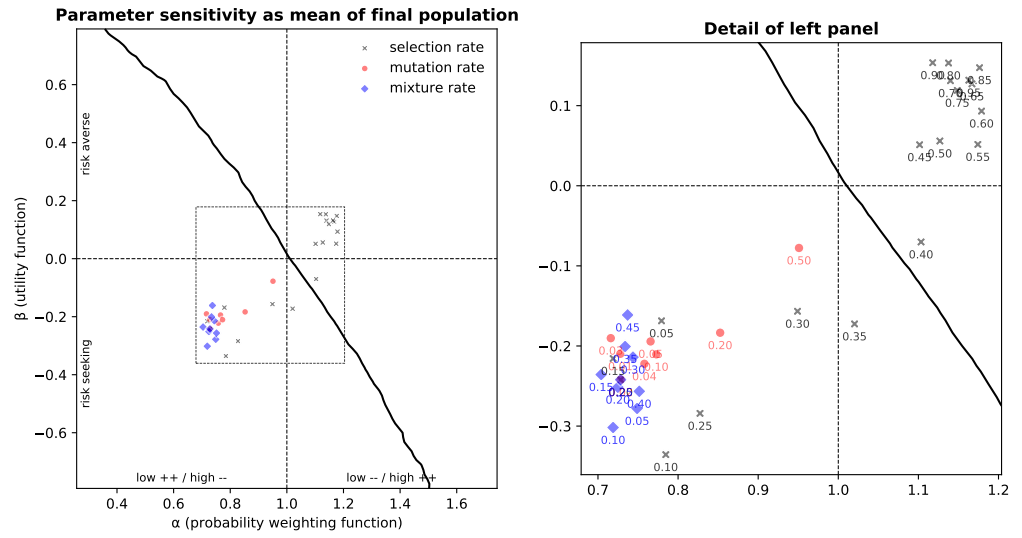

Figure 7: Parameter sensitivity. The exact shape of the final population's functions is dependent on the selection rate, mutation rate and selection rate. However, for a selection rate below $35 \%$, the general pattern remains the same: risk-seeking (convexity of the utility function) and overestimation of the small probabilities and underestimation of the high probabilities (inverted S-shaped probability weighting function). 
a

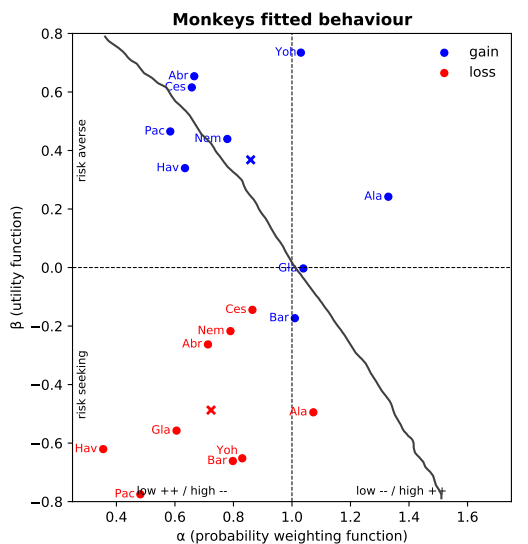

b

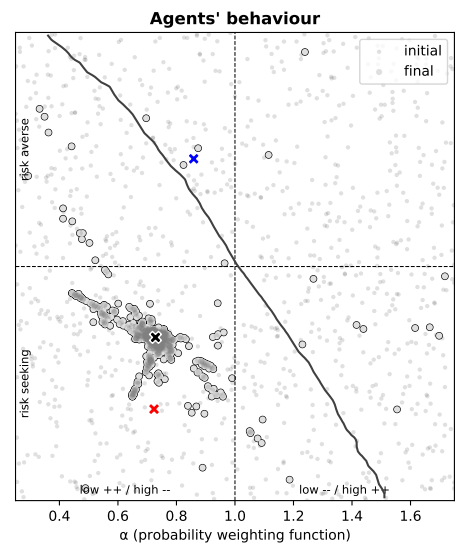

Figure 8: Comparison of monkeys' and agents' behaviour. (a) Using the result from the monkey's data fitting, we can represent the population for gain (blue) and loss (red) showing that the monkeys display a tendency to overestimate small probabilities and underestimate large probabilities (both in gain and loss domain) and that they are risk-averse in the gain domain but risk-seeking in the loss domain. Subjects 'Hav' and 'Gla' are rhesus monkeys. (b) Initial population and final population of agents. The mean behaviour (black cross) is closer to monkeys' mean behaviour in the loss domain (red cross) compared to monkey's mean behaviour in the gain domain (blue cross). It is to be noted that the exact final position depends on the selection, mutation, and mixture rate (see Figure 5). 


\begin{tabular}{ll}
\hline parameter name & value \\
\hline number of lotteries $N_{L}$ & 1000 \\
number of agents $N_{A}$ & 1000 \\
number of epochs $N_{E}$ & 1000 \\
number of trials $N_{T}$ & 100 \\
$w_{\alpha}$ & $x \rightarrow e^{-(-l n p)^{\alpha}}$ \\
$u_{\beta}$ & $x \rightarrow e^{1-\beta}$ \\
$\alpha_{\text {min }}, \alpha_{\max }$ & $0.25,1.75$ \\
$\beta_{\min }, \beta_{\max }$ & $-0.80,+0.80$ \\
$\gamma$ (selection rate) & 0.20 \\
$\delta$ (mutation rate) & 0.02 \\
$\epsilon$ (mixture rate) & 0.25 \\
PRNG seed used for all displayed results & 123 \\
\hline
\end{tabular}

Table 1: Parameters used in all simulations unless stated otherwise. 